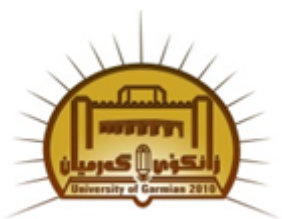

\title{
Molecular characterization of autochthonous grapevine (Vitis vinifera L.) cultivars in Kurdistan using simple sequence repeats (SSRs)
}

\author{
Asaad M. Mahmood ${ }^{1^{*}}$, Shaimaa H. Ali ${ }^{2}$, Shabaq M. Hawezy ${ }^{3}$ \\ ${ }^{1}$ Department of Biology, College of Education, University of Garmian \\ ${ }^{2}$ Scientific Research Center, College of Science, University of Duhok \\ ${ }^{3}$ Department of Horticulture, College of Agriculture, University of Salahaddin
}

\section{Article Info}

Received: July, 2019

Revised: September, 2019

Accepted: October, 2019

\section{Keywords}

Vitis vinifera, Germplasm characterization, Microsatellite, SSRs, Genetic diversity

\section{Corresponding Author} asaad@garmian.edu.krd

\begin{abstract}
Grapevine (Vitis vinifera L.) is one of the oldest and the most extensively grown fruit plants worldwide with regards to its great economic significance throughout the world. Northern part of Iraq (Kurdistan) is regarded as one of the regions in Iraq where grapevine plants have been taken into cultivation. Despite the economic importance of grapevine cultivars in this region, they have not been precisely classified and their genetic data is also limited. In the present study 12 simple sequence repeat (SSR) markers were used to evaluate the genetic diversity among 13 local grape cultivars, also determining their genetic relationships with four Eurpean ones (17 cultivars studied). Results showed that the observed number of alleles $(\mathrm{N})$ per locus varied from 3 to 9 with an average of 5.25 and the values of polymorphism information content (PIC) ranged from 0.28 to 0.776 . Despite the average value (0.1716) of observed heterozygosity (Ho) was less than the average level (0.6427) of expected heterozygosity $(\mathrm{He})$, results were relatively demonstrated a random union of gametes in the population. In addition, genetic similarity among respective cultivars ranged from 0.17 to 0.1 , indicating valuable genetic variation among studied cultivars. The analysis of UPGMA clustering demonstrated high genetic variation among those cultivars and grouped them into three main clusters. Findings from the present study might render striking information in determining local cultivars and in breeding management strategies that are of importance for genetic conservation and cultivar improvement.
\end{abstract}

\section{Introduction}

The frequently cultivated grapevine (Vitis vinifera $\mathrm{L}$.) is one of the most extensively grown fruit plants worldwide with regards to its great economic significance throughout the world (Riaz et al., 2018; Laucou et al., 2018). Although grapevine belongs to the Ampelidaceae family, it is recently classified under Vitaceae family, which includes 17 genera
(Schuck et al., 2014). The most important genus within this family is Vitis, the only genus to produce edible fruits (Dauob et al., 2018). It's believed that grapevines have a Caucasian origin and native to the Mediterranean and Central Asia (Khadivi et al., 2019). The most important genus within this family is Vitis, the only genus to produce edible fruits (Dauob et al., 2018). It's 
believed that grapevines have a Caucasian origin and native to the Mediterranean and Central Asia (Khadivi et al., 2019; Dauob et al., 2018). The cultivated grapevine (Vitis vinifera L.) has a long history of domestication and the Caucasian regions are believed to be within the primary center of this domestication (Rao et al., 2014; Khadivi et al., 2019). Then, the ancestor of cultivated grapevines has spread over the continents including Asia. Then, the ancestor of cultivated grapevines has spread over the continents including Asia (Dauob et al., 2018).

Currently, more than 9600 cultivars of the grape have been documented with almost 16,000 primitive names listed in the Vitis International Variety Catalogue. Several cultivars are difficult to be distinguished morphologically (Rao et al., 2014). The accurate discrimination and distinguishing of morphologically similar varieties/cultivars are difficult, which might lead to cultivar homonyms and/or synonyms (Nagaty and El-Din, 2011). Molecular techniques have been greatly solved such problems, which provide accurate and reliable identification of closely related genotypes (Basheer-Salimia and Mujahed, 2019).

To dates, many DNA-based approaches have been used for molecular identification and characterization of grape cultivars in the Mediterranean region (Karatas et al., 2019; Riaz et al., 2018). Among these molecular techniques, microsatellite markers (SSR) has received great attention due to its power to discriminate and unambiguously identify every individual through providing a unique genetic profile for each cultivar, inherited in a co-dominant Mendelian manner, not affected by environment, disease, or farming methods (Filippo et al., 2018).

Despite the importance of molecular identification of plants, it is clear that the information and knowledge about genetic diversity of fruits and particularly grapevine in Kurdistan region is limited. Consequently, it is of value to investigate aboriginal grape cultivars and genotypes from the respective domestication area and to determine their genetic relationships and diversities. Thus, in this study, the SSR markers are used to investigate the genetic diversity and phylogeny of grape cultivars in order to organize, describe, and characterize these valuable germplasms in Kurdistan region. This study will render striking information in breeding management strategies for genetic conservation and cultivar improvement in this region.

\section{Materials and Methods}

\section{Plant material}

All plant materials were collected from Agriculture Research Center in Erbil, a total of seventeen grape samples including 13 local (Rashmirre, Merani-Erbil, Zarg-Duhok, Rashmew, Bea-dank, Chav-ga, Kazhaw, Rashmirredriaj, Rashmirre-Duhok, Rashmirre-xr, Kamali, Sur-sham and Yaqwty) and 4 European (Baladysuree, Perlette, Rossi3, Slopee) cultivars were evaluated in the present study. Local cultivars are well adapted to Kurdistan's climatic conditions that might be used to establish new commercial orchards throughout the region as reference domestic cultivars. Other European cultivars were included to assess their genetic relationships with local cultivars.

\section{Molecular analysis}

Young leaves of respective cultivars were collected from each cultivar, $100 \mathrm{mg}$ of each sample was frozen with liquid nitrogen, and grinded into powder using Pistil and mortars. Following manufacturer's instructions, DNA was extracted from the powder of each cultivar using DNeasy® Plant Mini kit (Qiagen, UK). Extracted DNA were qualified and quantified using a NanoDrop 2000 spectrophotometer (Thermo Scientific, USA) and also Agarose gel electrophoresis. The PCR reaction mixture of 40 $\mu$ l contained $10 \mathrm{ng}(2 \mu \mathrm{l})$ of template, $20 \mu \mathrm{l} 2 \mathrm{X}$ BioMix PCR master mix (Bioline, UK,), $6 \mu \mathrm{l}$ $(10 \mathrm{pmol})$ of forward and reverse primer (German), $3 \mathrm{mmol} / \mathrm{L} \mathrm{MgCl}$, and 5.00\% DMSO. All amplifications were performed using a thermocycler (Eppendorf ${ }^{\mathrm{TM}}$ Mastercycler ${ }^{\mathrm{TM}}$ Nexus Thermal Cycler) with the following steps: 5 min at $95{ }^{\circ} \mathrm{C}$; 35 cycles at $95{ }^{\circ} \mathrm{C}$ for $30 \mathrm{~s}, 57$ $67{ }^{\circ} \mathrm{C}$ for $30 \mathrm{~s}, 72{ }^{\circ} \mathrm{C}$ for $1 \mathrm{~min} ; 72^{\circ} \mathrm{C}$ for 10 min. All PCR reactions were stored at $4{ }^{\circ} \mathrm{C}$. As described in table 1 , genomic samples were typed for 12 specific and different SSR loci. PCR reactions were run at 90 volts for 1-1.5 
hours in a horizontal gel electrophoresis unit (Fisher scientific, USA). The gel was visualized under a UV lamp and followed by capturing a clear picture using a Nikon digital camera.

\section{Data scorning and cluster analysis}

Gel electrophoresis images were used to score and analyze SSR-data. Number of alleles $(\mathrm{Na})$, allele size (bp), expected heterozygosity (He), mean of observed heterozygosity (Ho), Polymorphism information content (PIC), Major allele frequency (MAF), F(Null) null allele frequency estimate (NF) and $\mathrm{P}$-values, genetic distances and phylogenetic tree, all were analyzed and constructed using Power marker V3.0. Fig Tree V1.3.1 software was also used to visualize the phylogenetic tree of the studied samples (Rambaut, 2009).

\section{Results and Discussion}

Satisfactory amplification products of all the SSR primer pairs used were obtained and they were multiallelic. As shown in table 2, VMC5G1 locus showed the lowest number of different alleles (Na) (three alleles) and VMC1C10 locus observed the highest (nine alleles) with a total of 63 alleles considering all loci (in an average of 5.25 alleles). Previous studies on grapes confirmed that the VVS2 locus was given the highest number of alleles (Nunez et al., 2004; Şelli et al., 2007; Tangolar et al., 2009; Agar et al., 2012). The average number of different alleles in the present study was 5.25, which is relatively similar to the average number of alleles observed by previous studies (Emanuelli et al., 2013).

The estimated heterozygosity $(\mathrm{He})$ varied within a range between 0.3045 (VMC5G1) and 0.7993 (VMC1C10). In addition, the observed heterozygosity $(\mathrm{Ho})$ ranged from 0.00 (VMC1G3-2, VMC2E2, VMC2A9, VMC5G1, VRZAG79, VMC5E11 and VMC16D4) to 0.8824 (VMC2C10), indicating the highest level of genetic diversity within the respective germplasm for VMC2C10 locus (Table 2). In light of previous findings, wide diversities were found among clonally propagated, outbreeding and perennial species of Vitis vinifera (Hvarleva et al., 2004; Dzhambazova et al., 2009). The
VMC2C10 locus might remarkably involve high levels of heterozygosity in grapes.

The usefulness and importance of each microsatellite marker (SSR) for reliable distinction is assessed by PIC value (Martín et al., 2003). Table 2 shows that the calculated PIC values among all pairs of markers ranged from 0.2809 (VMC5G1) to 0.7766 (VMC1C10), followed by 0.7483 and 0.7430 (VMC1G3-2 and VMC2E2). According to (Martin et al. 2003), the PIC and $\mathrm{Ne}$ are estimators of usefulness of SSRs for cultivar distinction. In addition, the major allele frequency of major per locus was $0.2941-0.8235$, with an average of 0.4853 which is similar to findings reported by (Wei et al., 2014). It might be of adaptive significance to detect high proportion of rare alleles. The capture and preservation of such alleles and genotypes is a valuable objective of any conservation strategy (Park et al., 2009). Despite most SSR locus showed a significant heterozygote in all studied cultivars with $\mathrm{P}$ values ranged from $0.85-1.00(\mathrm{P}<0.001)$, some loci (VMC5E11 and VMC16D4) showed very low heterozygote results with $\mathrm{P}$-values of 1.00 $(\mathrm{P}<0.001)$. The excess of homozygotes for latter loci may be due to the presence of null alleles (Brookfield, 1996; Flajoulot et al., 2005).

Pairwise genetic similarities were also calculated using Dice's coefficient. As shown in table 3, results demonstrated genetic similarities among the grape cultivars from 0.17 (between Sur-sham and Rash-mew cultivars) to 1.00 (between Balady-suree and Bea-Dank, Kamali, Kazhaw, Merani-Erbil, Perlette, Rash-mew, Rashmirredriaj, Rashmirre-Duhok, Sur-sham, ZargDuhok). The genetic similarity in a grape germplasm in Turkey were ranged from 0.125 to 1.00 (Eyduran et al., 2016). Also, a range of 0.14-0.93 for the genetic similarity in grape germplasms of different Iranian grape cultivars were reported by (Khadivi et al., 2019).

Moreover, with the results obtained from the UPGMA cluster analysis, a dendrogram was constructed to clarify the genetic relationship between all the studied cultivars. The dendrogram revealed valuable genetic variation among studied cultivars, which grouped them into three main clusters (Fig. 1).

The first main cluster (blue color) consisted of six cultivars including Bea-dank, Kamali, 
Merani-Erbil, Rash-mew, Sur-sham and ZargDuhok. The second cluster (orange color) contained five cultivars namely, Rashmirredriaj, Rashmirre-Duhok, Rashmirre-xr, Kazhaw and Yaqwty. Also, four cultivars including Perlette, Rossi3, Slopeethe and Rashmirre were belonged to the third cluster (brown colour). Despite obtaining three main clusters, two other clusters (red and green colours) were determined and each of them contained only one cultivar (Balady-suree and Chav-ga respectively). Genotypic analysis of the studied grape germplasm at 12 nuclear microsatellite (SSR) loci demonstrated high levels of genetic variations. These variations might due to the variable environmental conditions of the origins of selected grapevines in Kurdistan region/North of Iraq. This result is similar to previous findings reported in different grapevine datasets, which are beneficial for breeders that they take advantage of these genetic variants in order to improve existing cultivars and create new varieties or accessions. (Martín et al., 2003; Grassi et al., 2006; Lopes et al., 2009; Pipia et al., 2012; Imazio et al., 2013; Khadivi et al., 2019).

According to the traditional and local nomenclature, it was expected to observe the Rashmirre cultivar with the other cultivars of Rashmirre (Rashmirre-driaj, Rashmirre-Duhok, and Rashmirre-xr). However, the presence of Rashmirre with Perlette, Rossi3 and Slopee (brown cluster) might be due to the wrong identification by farmers or local research centers based on its morphological characteristics. As stated above, it is difficult to accurately discriminate and distinguish cultivars that are morphologically very similar, which subsequently leads to cultivar homonyms (Nagaty and El-Din, 2011). This result might be counted as new a finding, suggesting further analysis to confirm the phylogenetic position of the Rashmiree cultivar.

In conclusion, the genetic evaluation of the studied cultivars observed high genetic variation values giving valuable information to determine relationships between local and European grape cultivars in the study area. The SSR data of this study is of importance to introduce worthy inputs contributing further grapevine selection and breeding strategies to protect and exploit the grapevine germplasm in the studied region.

\section{References}

1. Agar G, Yildirim N, Ercisli S, et al. (2012) Determination of genetic diversity of Vitis vinifera cv. Kabarcik populations from the Coruh Valley using SSR markers. Biochemical Genetics 50: 476-483.

2. Basheer-Salimia R and Mujahed A. (2019) Genetic diversity of grapevine (Vitis vinifera L.) as revealed by ISSR markers. Journal of Plant Biotechnology 46: 1-8.

3. Brookfield J. (1996) A simple new method for estimating null allele frequency from heterozygote deficiency. Molecular Ecology 5: 453-455.

4. Dauob R, Makhoul G and Mahfoud H. (2018) Genetic Diversity among Grapevine (Vitis vinifera L.) Cultivars of Tartous Province (Syria) using Microsatellite Markers. International Journal of Agriculture \& Environmental Science 5: 54-58.

5. Dzhambazova T, Tsvetkov I, Atanassov I, et al. (2009) Genetic diversity in native Bulgarian grapevine germplasm (Vitis vinifera L.) based on nuclear and chloroplast microsatellite polymorphisms. Vitis 48: 115-121.

6. Emanuelli F, Lorenzi S, Grzeskowiak L, et al. (2013) Genetic diversity and population structure assessed by SSR and SNP markers in a large germplasm collection of grape. BMC plant biology 13: 39.

7. Eyduran SP, Ercisli S, Akin M, et al. (2016) Genetic characterization of autochthonous grapevine cultivars from Eastern Turkey by simple sequence repeats (SSRs). Biotechnology \& Biotechnological Equipment 30: 26-31.

8. Filippo F, Elisabetta N, La MS, et al. (2018) First characterisation of minor and neglected Vitis vinifera L. cultivars from Mount Etna. Horticultural Science 45: 37-46.

9. Flajoulot S, Ronfort J, Baudouin $\mathrm{P}$, et al. (2005) Genetic diversity among alfalfa (Medicago sativa) cultivars coming from a breeding program, using SSR markers. 
Theoretical and Applied Genetics 111: 14201429.

10. Grassi F, Labra M, Imazio S, et al. (2006) Phylogeographical structure and conservation genetics of wild grapevine. Conservation Genetics 7: 837-845.

11. Hvarleva T, Rusanov K, Lefort F, et al. (2004) Genotyping of Bulgarian Vitis vinifera L. cultivars by microsatellite analysis. Vitisgeilweilerhof- 43: 27-34.

12. Imazio S, Maghradze D, De Lorenzis G, et al. (2013) From the cradle of grapevine domestication: molecular overview and description of Georgian grapevine (Vitis vinifera L.) germplasm. Tree Genetics \& Genomes 9: 641-658.

13. Karatas H, karaağaç E, karataş d, et al. (2019) Molecular analysis of grapevine germplasm by SSR (simple sequence repeats) in diyarbakir province, Turkey. Applied Ecology and Environmental Research 17: 3927-3939.

14. Khadivi A, Gismondi A and Canini A. (2019) Genetic characterization of Iranian grapes (Vitis vinifera L.) and their relationships with Italian ecotypes. Agroforestry Systems 93: 435-447.

15. Laucou V, Launay A, Bacilieri R, et al. (2018) Extended diversity analysis of cultivated grapevine Vitis vinifera with $10 \mathrm{~K}$ genomewide SNPs. PLoS One 13: 1-27. e0192540.

16. Lopes M, Mendonça D, Rodrigues dos Santos $M$, et al. (2009) New insights on the genetic basis of Portuguese grapevine and on grapevine domestication. Genome 52: 790800.

17. Martín J, Borrego J, Cabello F, et al. (2003) Characterization of Spanish grapevine cultivar diversity using sequence-tagged microsatellite site markers. Genome 46: 10-18.

18. Nagaty MA and El-Din E-AS. (2011) Molecular characterization and genetic relationships among some grape (Vitis vinifera L.) cultivars as revealed by RAPD and SSR markers. European Journal of Experimental Biology 1: 71-82.

19. Nunez Y, Fresno J, Torres V, et al. (2004) Practical use of microsatellite markers to manage Vitis vinifera germplasm: molecular identification of grapevine samples collected blindly in DO "El Bierzo"(Spain). The Journal of Horticultural Science and Biotechnology 79: 437-440.

20. Park Y-J, Lee JK and Kim N-S. (2009) Simple sequence repeat polymorphisms (SSRPs) for evaluation of molecular diversity and germplasm classification of minor crops. Molecules 14: 4546-4569.

21. Pipia I, Gogniashvili M, Tabidze V, et al. (2012) Plastid DNA sequence diversity in wild grapevine samples (Vitis vinifera subsp. sylvestris) from the Caucasus region. Vitis 51: 119-124.

22. Rambaut A. (2009) FigTree v1. 3.1: Tree figure drawing tool. Edinburgh, UK.

23. Rao V, Narayanaswamy $P$ and Murthy B. (2014) Genetic Diversity Analysis in Grape (Vitis vinifera L.) Germplasm using Microsatellite Markers. American International Journal of Research in Formal, Applied \& Natural Sciences 6: 12-18.

24. Riaz S, De Lorenzis G, Velasco D, et al. (2018) Genetic diversity analysis of cultivated and wild grapevine (Vitis vinifera L.) accessions around the Mediterranean basin and Central Asia. BMC Plant Biology 18: 137.

25. Schuck MR, Biasi LA, Moreira FM, et al. (2014) Use of microsatellite markers to assess the identity and genetic diversity of Vitis labrusca and Vitis rotundifolia cultivars. Acta Scientiarum. Agronomy 36: 301-308.

26. Şelli F, Bakir M, Inan G, et al. (2007) Simple sequence repeat-based assessment of genetic diversity in Dimrit and Gemre grapevine accessions from Turkey. Vitis 46: 182-187.

27. Tangolar SG, Soydam S, Bakir M, et al. (2009) Genetic analysis of grapevine cultivars from the eastern Mediterranean region of Turkey based on SSR Markers. Tarim Bilim Derg 15: 1-8.

28. Wei X, Wang L, Zhang Y, et al. (2014) Development of simple sequence repeat (SSR) markers of sesame (Sesamum indicum) from a genome survey. Molecules 19: 5150-5162. 
Table 1: Sequences of SSR primers used to characte rize grapevine cultivars in Kurdistan region

\begin{tabular}{|c|c|c|c|}
\hline NO. & LOCUS & FORWARD 5'--------3' & REVERS 5'--------3' \\
\hline 1. & VMC2B11 & AAGAAGGTGA CACCA GCGGA & CCCCTCACCCTGTTACCAAA \\
\hline 2. & VMC1G3-2 & GATAGTTACCATACTTA GTCGGA & ACTTAGCTTCAGAAGAAAATAGA \\
\hline 3. & VMC2E2 & CTTGTACACCCAA GATCTTCCA & TTAGTTAGTCATCTTCCACCCTCA \\
\hline 4. & $\mathrm{VMC1C10}$ & CACAGCTGTTCCAA GTCCCA & ACAAGCCTTCCGCCACTCTC \\
\hline 5. & VMC7A4 & TAAGGTGGATTA GTTTTGGGTC & AAACTCCAAAGCATCTGATTCT \\
\hline 6. & VMC4G6 & CCTTGAAGA GATGA GTTTGCTA & TATTTAACTTTGTGCCTCTGCT \\
\hline 7. & VMC2A9 & CAATCAAATCAGTTGATTGGACA & TTGGAAGATTATTTTGCTGGCT \\
\hline 8. & VMC5G1 & GAACTAAATGAAGCTGGAAAAGA & AAATTGCTTTGAATGAACACTG \\
\hline 9. & VRZAG79 & AGATTGTGGA GGA GGGAACAAACCG & TGCССССАТTTTCAAАСТСССТТСС \\
\hline 10. & VMC5E1 1 & ATTGGGTTGTATGATATTATTTT & TATTTATGTATTAGCATGCTTTT \\
\hline 11. & VMC16D4 & TAGAATACACAGGCCATATACAA & GGTTAGGATGCTTATA GAA GAA G \\
\hline 12. & $\mathrm{VMC} 2 \mathrm{C} 10$ & CCATGTTTCTATA GTTCTTCGGC & GATGTCCATATATTGGGTCCTG \\
\hline
\end{tabular}

Table 2: Number of alleles (Na), alle le size (bp), expected heterozygosity (He) and mean observed heterozygosity (Ho), Polymorphism information content (PIC), Major allele fre quency (MAF), F(Null) null alle le fre quency estimate (NF) and P-values in microsatellite loci of 17 cultivars of Vitis vinifera in Kurdistan region.

\begin{tabular}{l|cccccccc}
\multicolumn{1}{c}{ LOCUS } & NA & $\begin{array}{c}\text { ALLELE } \\
\text { SIZE }(\mathbf{B P})\end{array}$ & $\mathbf{( H e})$ & $\mathbf{( H o})$ & $\mathbf{P I C}$ & $\mathbf{M}_{\mathbf{A F}}$ & $\mathbf{N F}$ & $\boldsymbol{P}$-Val. \\
\hline VMC2B11 & 7 & $170-280$ & 0.6696 & 0.2353 & 0.6400 & 0.5294 & 0.5839 & 1.0000 \\
VMC1G3-2 & 6 & $170-290$ & 0.7820 & 0.0000 & 0.7483 & 0.2941 & 1.0000 & 1.0000 \\
VMC2E2 & 6 & $180-290$ & 0.7751 & 0.0000 & 0.7430 & 0.3529 & 1.0000 & 1.0000 \\
VMC1C10 & 9 & $190-300$ & 0.7993 & 0.4706 & 0.7766 & 0.3529 & 0.2773 & 0.9949 \\
VMC7A4 & 7 & $140-250$ & 0.7751 & 0.1176 & 0.7433 & 0.3235 & 0.7406 & 1.0000 \\
VMC4G6 & 4 & $130-190$ & 0.4637 & 0.3529 & 0.4253 & 0.7059 & 0.1045 & 0.9995 \\
VMC2A9 & 4 & $130-160$ & 0.5606 & 0.0000 & 0.4947 & 0.5882 & 0.9999 & 1.0000 \\
VMC5G1 & 3 & $130-180$ & 0.3045 & 0.0000 & 0.2809 & 0.8235 & 0.9828 & 1.0000 \\
VRZAG79 & 4 & $260-360$ & 0.5882 & 0.0000 & 0.5128 & 0.5294 & 0.9999 & 1.0000 \\
VMC5E11 & 4 & $200-260$ & 0.6367 & 0.0000 & 0.5694 & 0.4706 & 1.0000 & 1.0000 \\
VMC16D4 & 5 & $190-350$ & 0.6851 & 0.0000 & 0.6305 & 0.4118 & 1.0000 & 1.0000 \\
VMC2C10 & 4 & $160-240$ & 0.6730 & 0.8824 & 0.6133 & 0.4412 & -0.1792 & 0.8590 \\
AVERAGE & $\mathbf{5 . 2 5}$ & & $\mathbf{0 . 6 4 2 7}$ & $\mathbf{0 . 1 7 1 6}$ & $\mathbf{0 . 5 9 8 2}$ & $\mathbf{0 . 4 8 5 3}$ & &
\end{tabular}




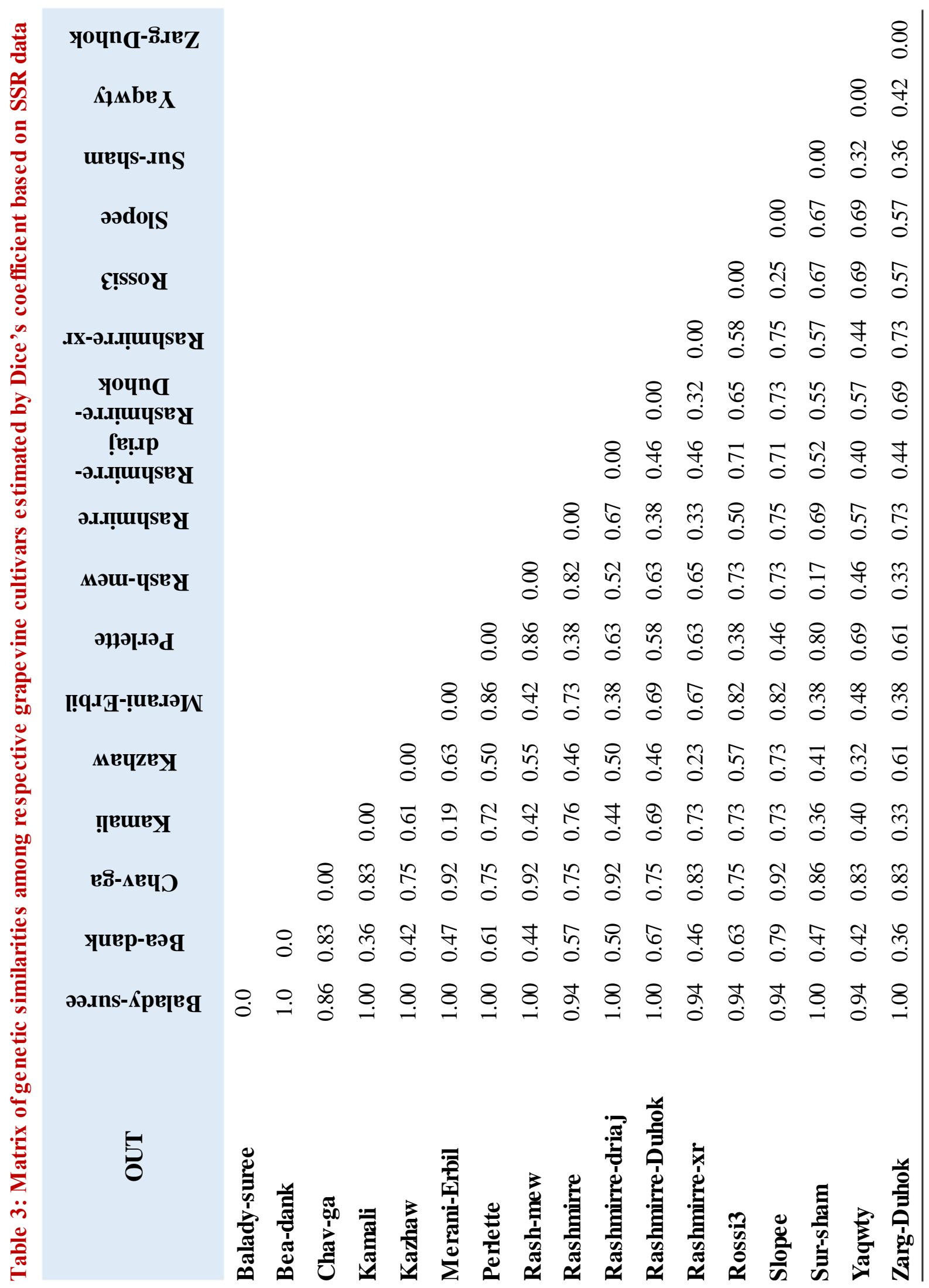




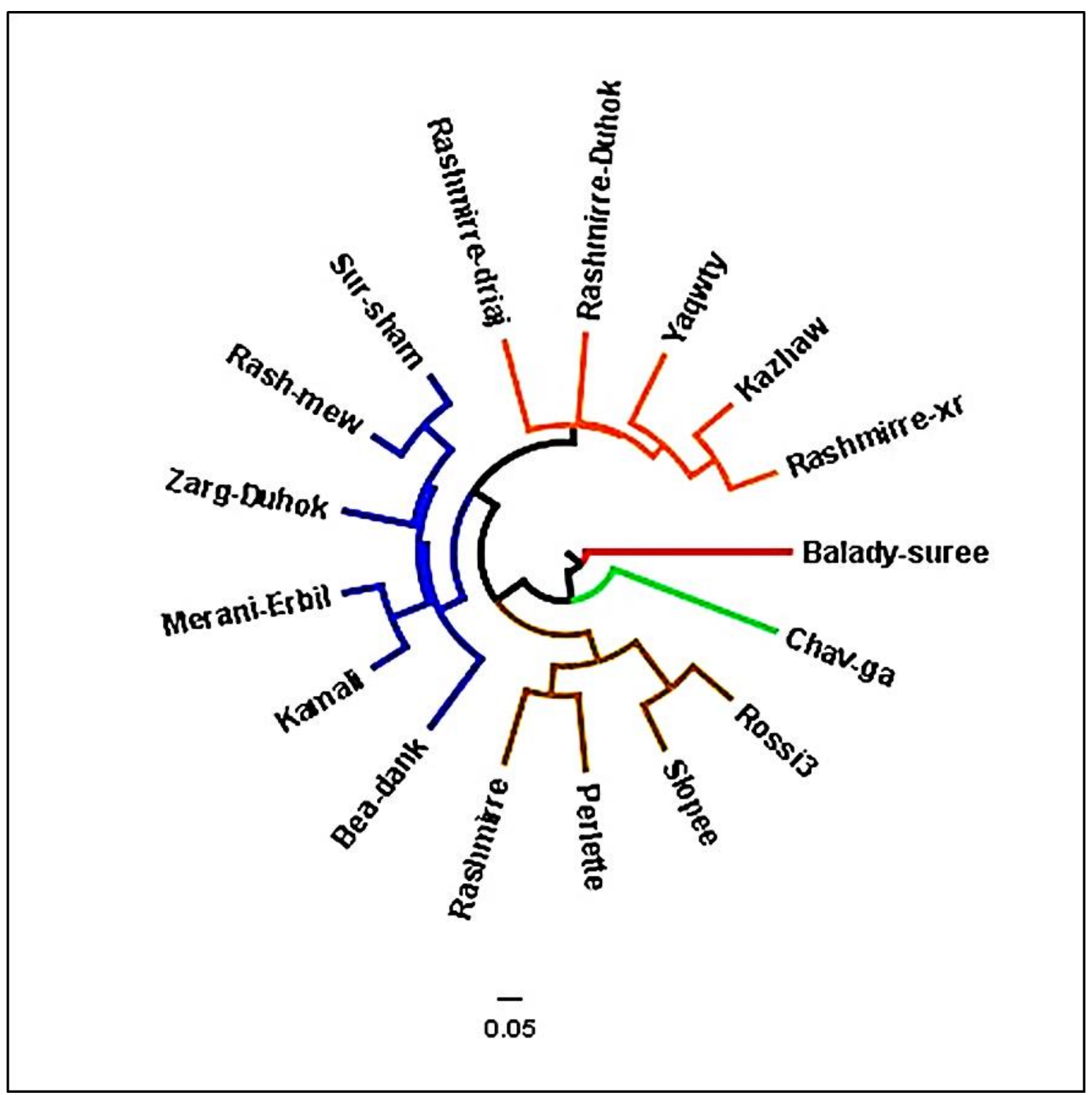

Figure 1: UPGMA dendrogram for the studied grape cultivars in Kurdistan region using Dice's coefficient based on SSR data 